\title{
Immunohistochemistry of a Proliferation Marker Ki67/MIB1 in Adrenocortical Carcinomas: Ki67/MIB1 Labeling Index Is a Predictor for Recurrence of Adrenocortical Carcinomas
}

\author{
RYO MORIMOTO, FUMITOSHI SATOH, OSAMU MURAKAMI, TAKASHI SUZUKI*, TAKAAKI ABE, \\ MASAYUKI TANEMOTO, MICHIAKI ABE, AKIRA URUNO, SHIGETO ISHIDOYA**, YOICHI ARAI**, \\ KAZUHIRO TAKAHASHI***, HIRONOBU SASANO\# AND SADAYOSHI ITO \\ Division of Nephrology, Endocrinology, and Vascular Medicine, Department of Medicine, Tohoku University Graduate School of \\ Medicine, Sendai 980-8574, Japan \\ *Department of Pathology, Tohoku University School of Health Sciences, Sendai 980-8574, Japan \\ **Department of Urology, Tohoku University Graduate School of Medicine, Sendai 980-8574, Japan \\ ***Department of Analytical Medical Technology, Tohoku University School of Health Sciences, Sendai 980-8574, Japan \\ \#Department of Pathology, Tohoku University Graduate School of Medicine, Sendai 980-8574, Japan
}

\begin{abstract}
Adrenocortical carcinoma (ACC) is a rare, highly malignant tumor. The aim of the present study is to evaluate the prognostic relevance of a proliferation marker Ki67/MIB1 by immunohistochemistry in 17 cases who underwent resections of the primary tumors and diagnosed to have ACC at Tohoku University Hospital based on the criteria of Weiss during the period from 1976 to 2005 . The follow-up periods ranged from 221 days to 10659 days (about 29 years) with the median of 1895 days. The median age at diagnosis was 46 years old, and the mean size of the primary tumors was $7.1 \mathrm{~cm}$ with the minimal of $3.5 \mathrm{~cm}$. Ki67/MIB1 labeling index (Ki67/MIB1LI) ranged from 1\% to 26\%. Kaplan-Meier analysis revealed that patients with Ki67/MIB1LI of 7\% or more were associated with significantly shortened disease-free survival $(P=0.0037)$. The evaluation with Weiss criteria revealed that the median score of Weiss criteria was five, and 13 patients $(76.5 \%)$ presented positive findings in the criteria of mitotic rate. The survival analysis with Weiss score showed that patients with the scores of 6 or more had both significantly shortened disease-free survival $(P=0.0001)$ and overall survival $(P=0.0063)$. The present study has suggested that Ki67/MIB1LI, as well as Weiss score, is a useful predictor for tumor recurrence after resection of the primary tumors in patients with ACC.
\end{abstract}

Key words: Adrenocortical carcinoma, Prognosis, Ki67

(Endocrine Journal 55: 49-55, 2008)

\begin{abstract}
ADRENOCORTICAL carcinoma (ACC) is a rare, highly malignant tumor with an annual incidence of approximately two cases per million population and overall five-year survival rate of $16-38 \%$ [1-3]. The prognosis of patients with ACC depends largely on surgical resectability [4]. An increasing number of

Received: May 9, 2007

Accepted: September 23, 2007

Correspondence to: Sadayoshi ITO, M.D., Ph.D., Division of Nephrology, Endocrinology, and Vascular Medicine, Department of Medicine, Tohoku University Graduate School of Medicine, 11 Seiryo-machi, Aoba-ku, Sendai 980-8574, Japan
\end{abstract}

adrenal tumors have been discovered because of technological advances in imaging modalities such as computed tomography (CT) and magnetic resonance imaging (MRI) [5], suggesting the possibility that an increasing number of smaller ACCs can be detected as adrenal incidentalomas. It is, therefore, important to diagnose ACC accurately and efficiently among these adrenal incidentalomas and to make an appropriate strategy for treatment.

The diagnosis of ACC has been made according to the criteria of Weiss $[6,7]$, which is a well-validated and established scoring system for a histopathological diagnosis of ACC $[1,8]$. ACC is documented to dem- 
onstrate high recurrence rate of up to $85 \%[1,9]$, and histological and molecular markers for prediction of tumor behavior are therefore considered to be one of major concerns. For example, in a series of 124 patients with ACC, Stojadinovic et al. revealed that as many as $84 \%$ of patients who underwent 'curative' resections of localized primary tumors demonstrated recurrences in their median follow-up period of 4.7 years [9]. The assessment of risk for future recurrences after resection of the primary tumors may therefore help to develop a rationale for adjuvant therapies.

To date, a number of molecular and histological markers have been studied to evaluate their efficacy to predict biological behaviors of ACC. A histological marker of Ki67/MIB1 labeling index (Ki67/MIB1LI) has been widely validated as a prognostic marker in various tumors $[10,11]$, and demonstrated to be effective in differentiating ACC from adrenal adenomas $[8,12-17]$. Its prognostic value to predict clinical outcome of ACC is, however, yet to be determined.

In the present study, we examined expression of Ki67/MIB1 by immunohistochemistry in 17 cases of ACC to evaluate the efficacy of this marker as a prognostic indicator for clinical courses following resections of the primary lesions.

\section{Materials and Methods}

\section{Patients, tissue specimens, and follow-up}

We reviewed records of 17 patients who underwent surgical resections of the primary lesions of ACC from 1976 to 2005 at Tohoku University Hospital, Sendai, Japan. Tissue specimens of ACC were obtained during surgery, and fixed in 10\% formalin and embedded in paraffin wax. The diagnosis of ACC was made by two pathologists (TS and HS) according to the criteria of Weiss $[6,7]$.

The follow-up period was started when the histopathological diagnosis of ACC was given, and terminated when a patient was deceased or at the end of 2005 when a patient was alive. Complete follow-up data were available for all patients. The following data were collected for each patient: demographics, localization and size of tumors evaluated by CT and/or MRI, tumor staging at initial presentation assessed by the Sullivan modification of the McFarlane system with TNM definitions [1, 18, 19], hormonal data at initial presentation, disease status at the end of followup, date of last follow-up, date and cause of death, and additional therapies including administration of mitotane. The resection of the primary tumor was considered to be curative in all cases except for one case (corresponding to case 1 in Table 1), which exhibited a metastatic lesion at lung at the time of the first surgery. Additional treatments, including mitotane regimen, were given at the discretion of physicians in charge of each patient's care. Written informed consents were obtained from all the patients, and this study was approved by the Ethics Committee of Tohoku University School of Medicine.

\section{Immunohistochemistry for Ki67/MIB1}

Immunohistochemistry for Ki67/MIB1 was performed using $3 \mu \mathrm{m}$ slices from paraffin-embedded specimens and Histofine immunostaining kit (Nichirei Co. Ltd., Tokyo, Japan), as previously reported [16]. The monoclonal antibody against Ki67 (MIB1) (Immunotech, Luminy, France) was used at optimal dilution of $1: 50$. Phosphate buffered saline $(0.01 \mathrm{~mol} /$ liter, $\mathrm{pH}$ 7.4) or normal mouse IgG was used instead of the primary antibody as negative controls, and no immunoreactivities were observed in these control sections.

Before counting, the areas for analysis were assessed by two of the authors (TS and RM). In all of the cases, 5 to 10 high power fields (400-folds) were selected, and at least 1000 cells were independently evaluated by two of the authors (TS and RM). The number of Ki67/ MIB1-positive cells per 100 adrenocortical cells was designated as the labeling index. The cases in which interobserver differences were more than $5 \%$ were reevaluated together by the two authors mentioned above using a multi-headed microscope. We obtained the mean of the values in the cases in which inter-observer differences were less than 5\%. Intra- and inter-observer differences were $3.7 \%$ and $4.2 \%$, respectively.

\section{Statistical analysis}

Overall survival (OS) was calculated from the date of diagnosis to the date of death from any cause. Patients alive at the end of the follow-up were censored. Disease-free survival (DFS) was calculated from the date of diagnosis to the date of recurrence diagnosed by $\mathrm{CT}$ and/or MRI findings. Patients without any recur- 


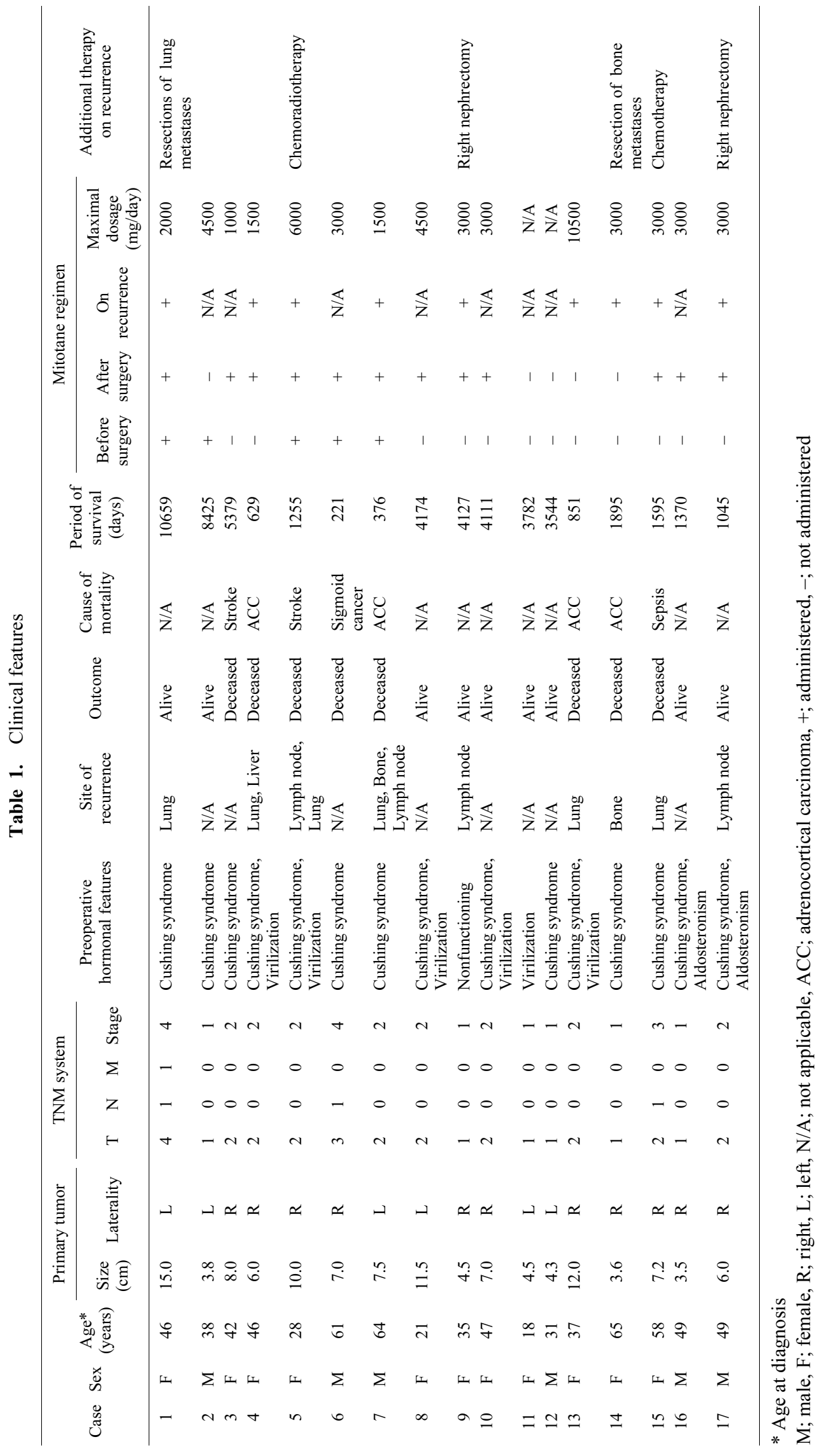


rence during the follow-up periods were considered as a censored case at the end of the follow-up or at the date of death from other causes than ACC.

All statistical analyses were carried out using JMP software version 6.0 (SAS Institute, Cary, NC, USA). Correlations of Ki67/MIB1LI with clinical variables were examined by $\chi^{2}$ test with a cross-table for ordinal and nominal variables, and evaluated using a one-way analysis of variance for continuous variables. Univariate survival analysis was conducted using the KaplanMeier method, and differences between survival curves were tested for significance using the log-rank test. The 17 patients were divided into two groups following the cut-off values of Ki67/MIB1LI and score of Weiss criteria, respectively. The cut-off values were determined by the decision tree/partition platform provided by JMP software version 6.0. $P$ values less than 0.05 were considered statistically significant.

\section{Results}

\section{Clinical findings}

The follow-up periods ranged from 221 days to 10659 days (about 29 years) with the median of 1895 days. The clinical features of the 17 patients $(6$ males and 11 females) are summarized in Table 1 . The median age at diagnosis was 46 years old, and the mean size of the primary tumors was $7.1 \mathrm{~cm}$ with the minimal of $3.5 \mathrm{~cm}$. In 15 patients $(88.2 \%)$, mitotane therapy was initiated at some point of their follow-up periods, and the dosage varied between 1 and $10.5 \mathrm{~g} /$ day. Following removal of the primary lesions, resection of locally recurrent lesions with nephrectomy was performed in two patients (case 9 and 17), and metastatic lesions of lung and bones were resected in patients corresponding to case 1 and 14, respectively. Clinical variables of sex, age at diagnosis, stage at initial presentation, size, laterality, and hormonal function of the primary tumors showed no significant correlations with Ki67/MIB1LI, as shown in Table 2.

\section{Analysis of Ki67/MIB1LI and Weiss score}

The analysis of the 17 patients with Ki67/MIB1LI and Weiss score is shown in Table 3. Ki67/MIB1LI ranged from 1 to $26 \%$ with the mean of $7.9 \%$. The representative presentations of Ki67/MIB1-positive nuclei
Table 2. Correlations between clinical variables and Ki67/ MIB1 labeling index

\begin{tabular}{lccc}
\hline & \multicolumn{2}{c}{ Ki67/MIB1 labeling index } & \multirow{2}{*}{$P$} \\
\cline { 2 - 3 } & $<7(\mathrm{n}=11)$ & $\geq 7(\mathrm{n}=6)$ & \\
\hline Sex & & & 0.9006 \\
$\quad$ Male & $4(36.4 \%)$ & $2(33.3 \%)$ & \\
Female & $7(63.6 \%)$ & $4(66.7 \%)$ & \\
Age (years)* & $39.6[30.9-48.4]$ & $49.8[38.0-61.7]$ & 0.1606 \\
TNM stage & & & 0.3992 \\
I & $4(36.4 \%)$ & $2(33.3 \%)$ & \\
II & $5(45.5 \%)$ & $3(50.0 \%)$ & \\
III & $0(0 \%)$ & $1(16.7 \%)$ & \\
IV & $2(18.1 \%)$ & $0(0 \%)$ & \\
Size (cm)* & $7.5[5.3-9.7]$ & $6.5[3.5-9.4]$ & 0.5519 \\
Laterality & & & 0.2352 \\
Right & $6(54.5 \%)$ & $5(83.3 \%)$ & \\
$\quad$ Left & $5(45.5 \%)$ & $1(16.7 \%)$ & \\
Hormonal function & & & 0.1628 \\
Functioning & $11(100 \%)$ & $5(83.3 \%)$ & \\
Nonfunctioning & $0(0 \%)$ & $1(16.7 \%)$ & \\
\hline
\end{tabular}

* Data were presented as mean, and confidence intervals of $95 \%$ were shown in brackets. All other values represented the number of cases, and the percentages compared with the total number were shown in parentheses.

are shown in Figure 1B, 1D, and 1F. The evaluation with Weiss criteria revealed that the median score of Weiss criteria was five, and 13 patients $(76.5 \%)$ presented positive findings in the criteria of mitotic rate.

The cut-off value, 7\% of Ki67/MIB1LI, which was determined by the decision tree/partition platform, was used to divide the 17 patients into two groups (Fig. 2A, 2B). Univariate survival analysis revealed that Ki67/ MIB1LI equal to or greater than $7 \%$ was associated with significantly shortened DFS $(P=0.0037$; Fig. 2A), whereas it was not associated with shortened OS $(P=0.1615$; Fig. 2B). Univariate survival analysis with Weiss score showed that patients with the scores of 6 or more had both significantly shortened DFS $(P=0.0001$; Fig. $2 \mathrm{C})$ and $\mathrm{OS}(P=0.0063$; Fig. 2D).

\section{Discussion}

The present study demonstrated that Ki67/MIB1LI may have prognostic relevance to predict recurrences after resection of the primary tumors. Immunohistochemical analysis with Ki67/MIB1 has been widely performed in various human malignancies, and its role both as a diagnostic tool and as a prognostic factor has 
Table 3. Pathological features

\begin{tabular}{|c|c|c|c|c|c|c|c|c|c|c|c|}
\hline \multirow[b]{2}{*}{ case } & \multirow{2}{*}{$\begin{array}{c}\text { Ki67/MIB1 } \\
\text { LI (\%) }\end{array}$} & \multirow{2}{*}{$\begin{array}{l}\text { Weiss } \\
\text { score }\end{array}$} & \multicolumn{9}{|c|}{ Weiss criteria } \\
\hline & & & $\begin{array}{l}\text { nuclear } \\
\text { atypia }\end{array}$ & $\begin{array}{l}\text { mitotic } \\
\text { rate }\end{array}$ & $\begin{array}{l}\text { atypical } \\
\text { mitosis }\end{array}$ & $\begin{array}{c}\text { clear cells } \\
\leq 25 \%\end{array}$ & $\begin{array}{c}\text { diffuse } \\
\text { architecture }\end{array}$ & necrosis & $\begin{array}{l}\text { venous } \\
\text { invasion }\end{array}$ & $\begin{array}{c}\text { sinusoidal } \\
\text { invasion }\end{array}$ & $\begin{array}{l}\text { capsular } \\
\text { invasion }\end{array}$ \\
\hline 1 & 6 & 5 & 1 & 1 & 0 & 1 & 1 & 1 & 0 & 0 & 0 \\
\hline 2 & 2 & 3 & 1 & 0 & 0 & 0 & 1 & 0 & 0 & 0 & 1 \\
\hline 3 & 3 & 4 & 1 & 1 & 0 & 0 & 1 & 0 & 0 & 0 & 1 \\
\hline 4 & 5 & 7 & 1 & 1 & 0 & 1 & 1 & 1 & 1 & 0 & 1 \\
\hline 5 & 14 & 5 & 0 & 1 & 0 & 1 & 1 & 1 & 0 & 0 & 1 \\
\hline 6 & 4 & 6 & 1 & 0 & 0 & 1 & 1 & 1 & 0 & 1 & 1 \\
\hline 7 & 21 & 7 & 0 & 1 & 1 & 1 & 1 & 1 & 1 & 0 & 1 \\
\hline 8 & 4 & 5 & 1 & 1 & 0 & 1 & 1 & 0 & 0 & 1 & 0 \\
\hline 9 & 8 & 6 & 1 & 1 & 0 & 1 & 1 & 1 & 0 & 0 & 1 \\
\hline 10 & 2 & 4 & 1 & 1 & 0 & 1 & 1 & 0 & 0 & 0 & 0 \\
\hline 11 & 1 & 4 & 1 & 0 & 0 & 1 & 1 & 0 & 0 & 0 & 1 \\
\hline 12 & 3 & 4 & 1 & 0 & 0 & 1 & 1 & 0 & 1 & 0 & 0 \\
\hline 13 & 5 & 8 & 1 & 1 & 0 & 1 & 1 & 1 & 1 & 1 & 1 \\
\hline 14 & 19 & 5 & 0 & 1 & 1 & 1 & 1 & 0 & 0 & 0 & 1 \\
\hline 15 & 7 & 6 & 1 & 1 & 0 & 1 & 1 & 1 & 0 & 0 & 1 \\
\hline 16 & 5 & 4 & 1 & 1 & 0 & 0 & 1 & 0 & 0 & 0 & 1 \\
\hline 17 & 26 & 6 & 1 & 1 & 0 & 1 & 1 & 0 & 0 & 1 & 1 \\
\hline
\end{tabular}

In the columns of Weiss criteria, 0 and 1 signify negative and positive findings for each criterion, respectively. Ki67/MIB1LI; Ki67/ MIB1 labeling index
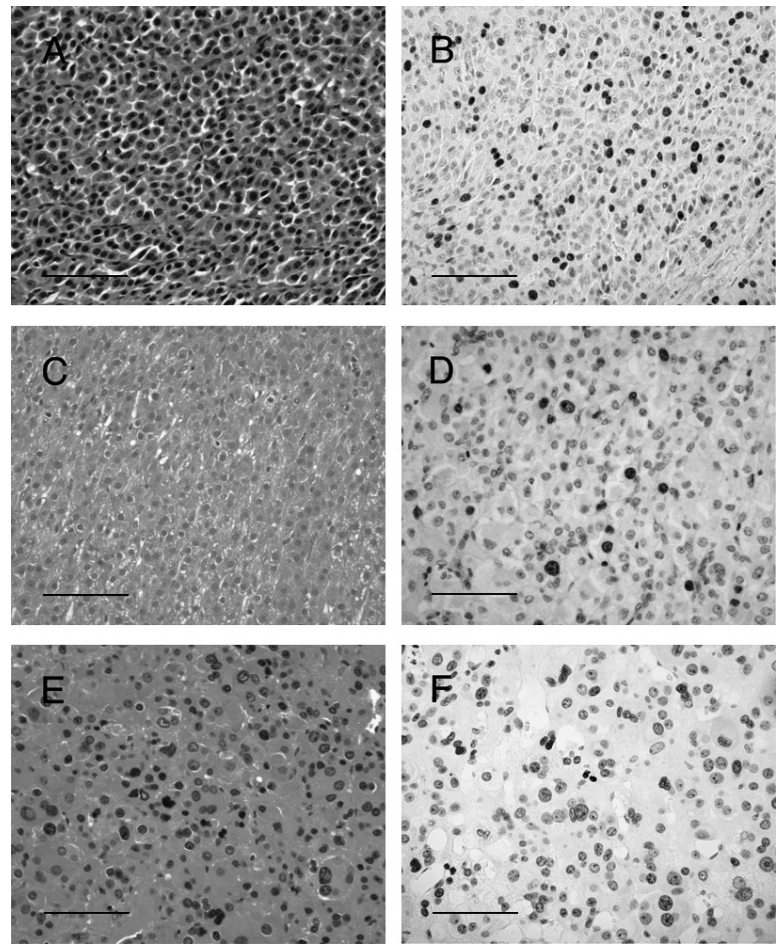

Fig. 1. Representative presentations of Ki67/MIB1-positive adrenocortical carcinoma cells. A and B; Case 17 showed high Ki67/MIB1 labeling index (Ki67/MIB1LI) of 26\%. C and D; Case 9 with intermediate Ki67/MIB1LI of $8 \%$. E and F; Case 11 with low Ki67/MIB1LI of $1 \%$. $(\mathrm{A}, \mathrm{C}, \mathrm{E})$ hematoxylin-eosin staining and $(\mathrm{B}, \mathrm{D}, \mathrm{F}) \mathrm{im}-$ munohistochemistry for Ki67/MIB1. Bars, $100 \mu \mathrm{m}$. been well established $[10,11]$. In human ACC, a high mitotic rate has been reported to be a significant and independent marker of poor clinical courses [18], and Ki67/MIB1LI was significantly related to mitotic count [12] and other parameters of cell proliferation such as expression of DNA topoisomerase type IIa [14] and p27 [16]. Previous studies including ours reported that the cut-off values of Ki67/MIB1LI between $2.5 \%$ and $5.0 \%$ reliably differentiated ACC from adrenal adenomas $[8,12-17,20]$. The information on the role of Ki67/MIB1LI as a predictor of clinical courses was limited, however, in patients with ACC [9, 21].

McNicol et al. reported that patients with Ki67/ MIB1LI of over 3\% had significantly shortened DFS, and that stratification with Ki67/MIB1LI of 3\% showed no significant differences in overall survival [21]. In contrast to the present study, they showed no clear reason for cut-off value of Ki67/MIB1LI 3\%, and included 13 patients who underwent subtotal resections and 2 patients without indication for resection of the primary tumors [21]. The report by McNicol et al. [21] and the present study commonly showed, however, that survival analysis with Ki67/MIB1LI was useful in predicting the duration of relapse-free state, but not the duration of survival. This may be due to the fact that expression of Ki67/MIB1 reflects only the proliferating capability of tumors $[12,14,16]$, but not other 
A

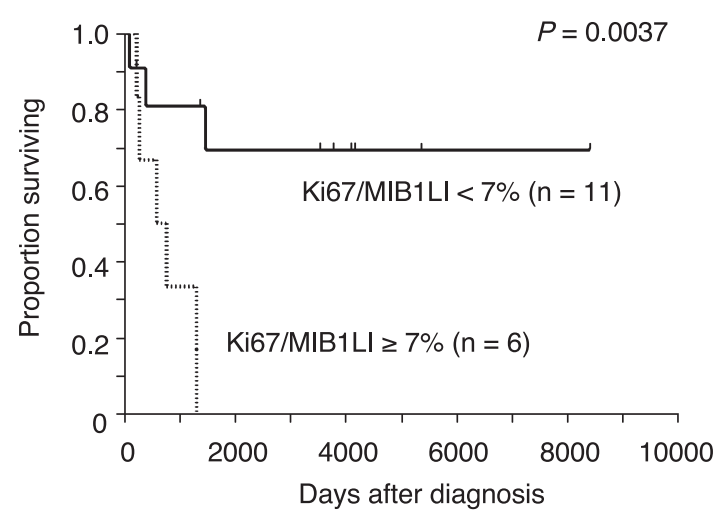

B

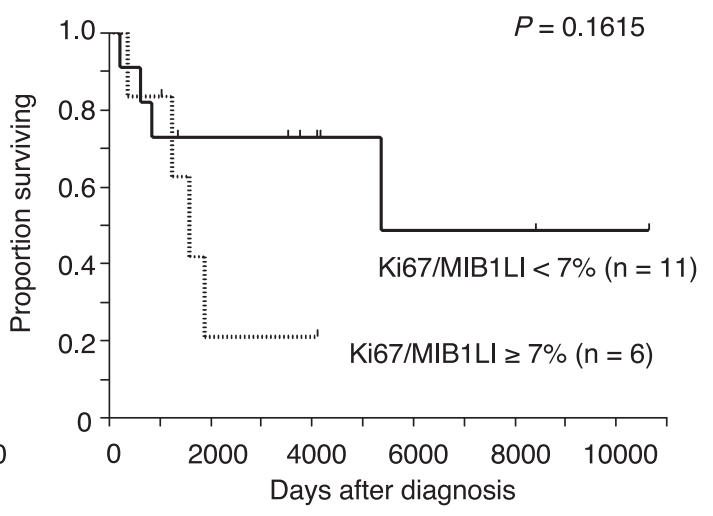

D

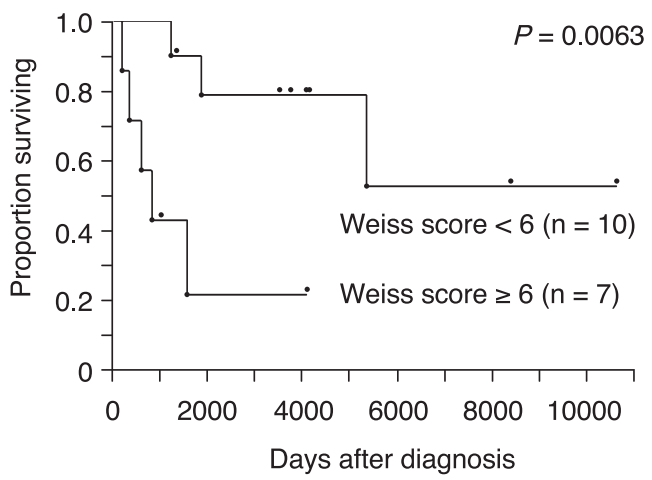

Fig. 2. Kaplan-Meier analysis of survival. A and C; Disease-free survival stratified by Ki67/MIB1 labeling index and Weiss score, respectively. B and D; Overall survival stratified by Ki67/MIB1 labeling index and Weiss score, respectively. All curves were examined by log-rank test for significant differences. Ki67/MIB1LI, Ki67/MIB1 labeling index.

properties, such as their transformative, invasive, and metastasizing abilities, all of which might determine the biological behaviors of these tumors and overall prognosis of patients. Furthermore, Stojadinovic et al. showed no significant differences in disease-specific survival when 31 patients were stratified according to the cut-off value of Ki67/MIB1LI 5\% [9]. Another finding shown in the present study is that the survival analysis with score of Weiss criteria was effective in predicting overall survival, probably because analysis with Weiss criteria includes not only proliferating ability but also other malignant potentials of invasion, atypia of tumor cells, and so forth.

The present study included a limited number of patients who were diagnosed and followed up at a single institution. Further prospective studies with larger number of cohorts consisting of multiple institutions are therefore required to validate the usefulness of Ki67/MIB1LI as a prognostic indicator. In addition, 15 out of the 17 patients had mitotane, and the time to initiate and/or terminate mitotane regimens and the dosages were arbitrarily determined in the present study. We could not exclude a possibility that mitotane regimen affected the clinical courses of these patients regardless of the pathological status of Ki67/MIB1LI and Weiss scores.

In conclusion, the present study has suggested that Ki67/MIB1LI, as well as Weiss score, has prognostic relevance to predict tumor recurrence. High levels of Ki67/MIB1LI may suggest progressive diseases rather than indolent clinical courses after resection of the primary tumors, and may justify aggressive adjuvant therapies to such patients. 


\section{Acknowledgments}

We wish to thank all the health care professionals who dedicated their expertise to the care of the patients included in the present study.

\section{References}

1. Allolio B, Fassnacht M (2006) Clinical review: adrenocortical carcinoma: clinical update. J Clin Endocrinol Metab 91: 2027-2037.

2. Dackiw AP, Lee JE, Gagel RF, Evans DB (2001) Adrenal cortical carcinoma. World J Surg 25: 914-926.

3. Wajchenberg BL, Albergaria Pereira MA, Medonca $\mathrm{BB}$, Latronico AC, Campos Carneiro P, Alves VA, Zerbini MC, Liberman B, Carlos Gomes G, Kirschner MA (2000) Adrenocortical carcinoma: clinical and laboratory observations. Cancer 88: 711-736.

4. Allolio B, Hahner S, Weismann D, Fassnacht M (2004) Management of adrenocortical carcinoma. Clin Endocrinol (Oxf) 60: 273-287.

5. Herrera MF, Grant CS, van Heerden JA, Sheedy PF, Ilstrup DM (1991) Incidentally discovered adrenal tumors: an institutional perspective. Surgery 110: 10141021.

6. Weiss LM (1984) Comparative histologic study of 43 metastasizing and nonmetastasizing adrenocortical tumors. Am J Surg Pathol 8: 163-169.

7. Weiss LM, Medeiros LJ, Vickery AL Jr (1989) Pathologic features of prognostic significance in adrenocortical carcinoma. Am J Surg Pathol 13: 202-206.

8. Sasano H, Suzuki T, Moriya T (2001) Discerning malignancy in resected adrenocortical neoplasms. Endocr Pathol 12: 397-406.

9. Stojadinovic A, Ghossein RA, Hoos A, Nissan A, Marshall D, Dudas M, Cordon-Cardo C, Jaques DP, Brennan MF (2002) Adrenocortical carcinoma: clinical, morphologic, and molecular characterization. $J$ Clin Oncol 20: 941-950.

10. Scholzen T, Gerdes J (2000) The Ki-67 protein: from the known and the unknown. J Cell Physiol 182: 311322.

11. Brown DC, Gatter KC (2002) Ki67 protein: the immaculate deception? Histopathology 40: 2-11.

12. Goldblum JR, Shannon R, Kaldjian EP, Thiny M, Davenport R, Thompson N, Lloyd RV (1993) Immunohistochemical assessment of proliferative activity in adrenocortical neoplasms. Mod Pathol 6: 663-668.

13. Sasano H, Imatani A, Shizawa S, Suzuki T, Nagura H (1995) Cell proliferation and apoptosis in normal and pathologic human adrenal. Mod Pathol 8: 11-17.

14. Iino K, Sasano H, Yabuki N, Oki Y, Kikuchi A, Yoshimi T, Nagura H (1997) DNA topoisomerase II alpha and Ki-67 in human adrenocortical neoplasms: a possible marker of differentiation between adenomas and carcinomas. Mod Pathol 10: 901-907.

15. Vargas MP, Vargas HI, Kleiner DE, Merino MJ (1997) Adrenocortical neoplasms: role of prognostic markers MIB-1, P53, and RB. Am J Surg Pathol 21: 556-562.

16. Nakazumi H, Sasano H, Iino K, Ohashi Y, Orikasa S (1998) Expression of cell cycle inhibitor p27 and Ki-67 in human adrenocortical neoplasms. Mod Pathol 11: 1165-1170.

17. Wachenfeld C, Beuschlein F, Zwermann O, Mora P, Fassnacht M, Allolio B, Reincke M (2001) Discerning malignancy in adrenocortical tumors: are molecular markers useful? Eur J Endocrinol 145: 335-341.

18. Schteingart DE, Doherty GM, Gauger PG, Giordano TJ, Hammer GD, Korobkin M, Worden FP (2005) Management of patients with adrenal cancer: recommendations of an international consensus conference. Endocr Relat Cancer 12: 667-680.

19. Norton JA (2005) Adrenal tumors. In: DeVita VT Jr, Hellman S, Rosenberg SA (eds) Cancer: principles and practice of oncology. 7th ed. Lippincott Williams \& Wilkins, Philadelphia: 1528-1539.

20. Schmitt A, Saremaslani P, Schmid S, Rousson V, Montani M, Schmid DM, Heitz PU, Komminoth P, Perren A (2006) IGFII and MIB1 immunohistochemistry is helpful for the differentiation of benign from malignant adrenocortical tumours. Histopathology 49: 298307.

21. McNicol AM, Struthers AL, Nolan CE, Hermans J, Haak HR (1997) Proliferation in adrenocortical tumors: correlation with clinical outcome and p53 Status. Endocr Pathol 8: 29-36. 